\title{
Openness about animal research increases public support
}

\author{
Science engagement can be a daunting prospect. This is especially true for scientists whose work involves animal \\ models, and particularly nonhuman primates. Here, we show that openly explaining our rationale for our neuroscience \\ work involving nonhuman primates - and the legal and ethical regulations that govern animal experimentation - \\ increased public support and understanding, which is crucial for this essential research to continue.
}

\section{Juan Carlos Mendez, Brook A. L. Perry, Rhyanne J. Heppenstall, Stuart Mason and Anna S. Mitchell}

I he use of animals in neuroscientific research has been essential to our current understanding of brain function, as well as for the development of therapies for neurological illnesses. Biomedical experimental findings on nonhuman primates (NHPs) are particularly transferable to humans, owing to their similar physiology and the anatomical resemblance of their brains to ours ${ }^{1}$. Although neuroscience has seen enormous advances since the middle of the twentieth century, research on animals (including NHPs) is still irreplaceable and support from policy makers and the public is crucial for its continuation. Thus, neuroscientists, supported by their funders and research institutions, need to keep raising awareness about the importance of their findings in animal models in creative and accessible ways.

Recognition of the need for transparency among researchers working with animals is increasing. In the UK, signatories of the 2014 Concordat on Openness on Animal Research committed to proactively and clearly communicate their research on animals with the media and the public ${ }^{2}$. This document has been signed by more than 120 research organizations, including our institution (University of Oxford) and our funders (The Wellcome Trust), and it has inspired countries worldwide to commit to similar agreements. An example of an effort along these lines is the creation of resources such as the Lab Animal Tour ${ }^{3}$, an interactive website with videos through which people can virtually access the animal facilities of four different research institutes of the UK, including the primate facility at the University of Oxford. The aims of this positive shift are to assuage the public on the ethical, moral and scientific justification for animal research, and to engage with regulators on the importance of biomedical research and the necessity for ensuring international collaboration ${ }^{4}$. Perhaps as paramount, these efforts should also inform about the scientists' work toward the humane treatment of NHPs.

In Europe and the USA, scientists working with animals must comply with strict laws that ensure animal welfare remains a priority ${ }^{4,5}$. In the UK, any project involving NHP experiments must first undergo extensive ethical approval to be granted a license by the Home Office Secretary of State and is then continuously scrutinized. One of the guiding principles behind these animal research laws is adherence to the 3Rs: proposed in 1959 in the UK and subsequently adopted internationally, the $3 \mathrm{R}$ principles advocate for replacement (avoiding the use of animals if alternatives exist), reduction (minimizing the number of animals used) and refinement (methods that minimize suffering and improve welfare). However, the public seems to be unaware of most of these efforts: a poll conducted by IPSOS Mori (https://www. ipsos.com $/)^{6}$ every 2 to 3 years to assess public attitudes toward animal research has consistently shown that less than $5 \%$ of the public is aware of the 3Rs. Similarly, this poll estimated that $38 \%$ of the population believe that cosmetic research on animals is legal in the UK, even though it has been banned since 1998 (and across the EU since 2013). Still, a recent petition submitted to the UK Government and Parliament ${ }^{7}$ asking 'to ban all UK animal testing, including for the development of cosmetics, household products and medicines' reached more than 235,000 signatures. Perhaps this widespread misinformation is behind the finding by the Pew Research Center ${ }^{8}$ that, whereas $89 \%$ of the community connected to the American Association for the Advancement of Science (AAAS) supports animal research, only $47 \%$ of the interviewed adults support it. Importantly, by the time this Comment is published, the Swiss government ${ }^{9}$ will have submitted to public vote, in February 2022, an initiative seeking to ban all animal experimentation in the country for the fourth time. Thus, despite scientists and governments working continuously toward improving the standards of animal research, there is still strong opposition to it.

These facts imply that outreach efforts from scientists working with animals are still not reaching wide sectors of the public ${ }^{10}$, a notion reinforced by the finding that the characteristic that is most commonly associated with animal research organizations is that they are secretive ${ }^{6}$. In a way, this is understandable: there is a history of harassment and aggression toward researchers using NHPs that could deter many from being open about their work $^{11}$. Nevertheless, governments have passed acts (such as the 2006 Animal Enterprise Terrorism Act in the USA) to protect researchers, and extreme violence against them is in decline ${ }^{2}$. Another reason for scientists' reluctance to engage with the public could be the common belief that participating in scientific outreach does not bring any benefit to their career ${ }^{12}$. After all, there is little evidence that public engagement actually yields a positive impact ${ }^{13}$, particularly in the field of animal research ${ }^{14-16}$. Thus, if neuroscientists working with NHPs are to be encouraged to speak openly about their work, it is crucial to assess whether reaching out to the public is fruitful and ultimately benefits both the public and the scientists.

Periodical polls, such as those by IPSOS Mori $^{6}$ and the Pew Research Center ${ }^{8}$, normally collect the views of a large population sample (1,011 and 2,002 adult individuals, respectively, in their latest editions) over several days or weeks, with individuals in the sample changing every time the study is repeated. Thus, although these polls provide important insights about changes in opinion among the public throughout the years, it is difficult to gauge from them which factors led to which changes. For this, the effect of 
Question 1: How well informed do you feel about animal research in the UK?

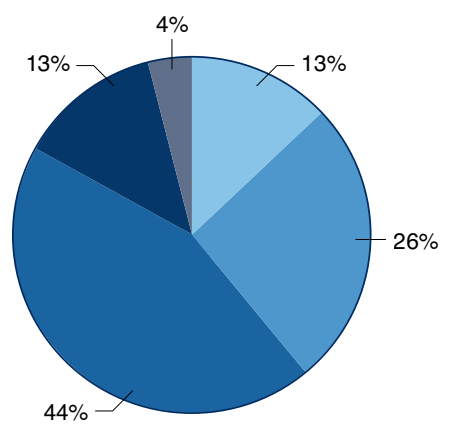

Very well informed

Fairly well informed

Not well informed

Not at all well informed

Don't know

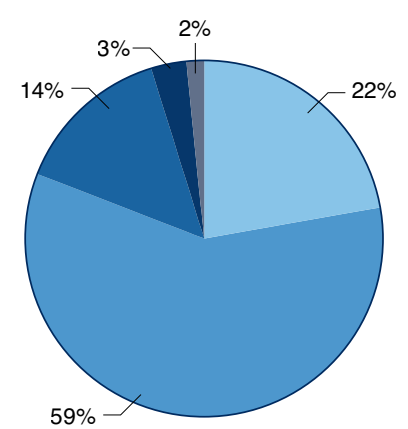

Question 2: How do you feel about animal research?

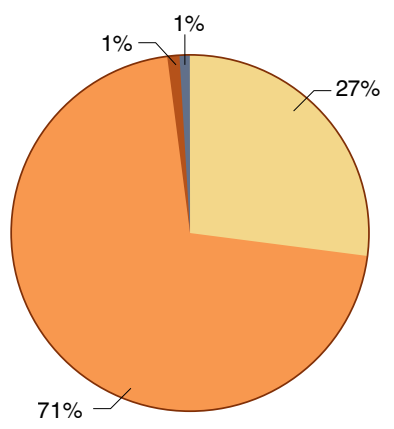

Animal research is necessary but cruel Animal research is necessary and can be done without cruelty

Animals should never be used in research

Don't know

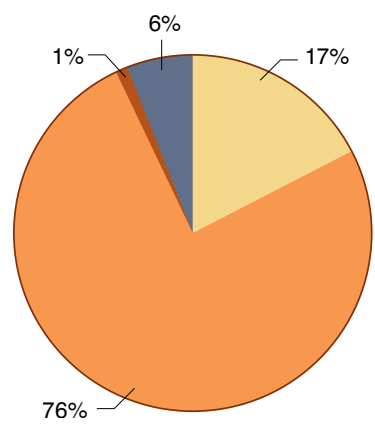

Question 3 (late poll only): Did the presentation help to change your perception of animal research?

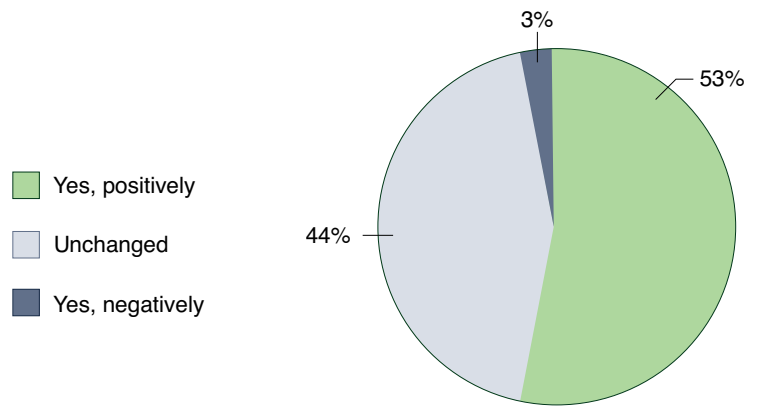

Fig. 1 | Effects of science engagement on perspectives on animal research. Total per cent results of the online surveys completed before (early poll) and immediately after (late poll) our online science engagement event 'Behind the Scenes of a non-human primate research lab', attended by people over the age of 13 years from the IF Oxford Science and Ideas Festival 2020 and the British Neuroscience Association 'Bring Your Own Brain' 2021 Festival of Neuroscience.

individual variables needs to be evaluated independently and, ideally, in the same sample. A few studies have analyzed how different informative strategies affect support for behavioral research experiments from visitors to primate zoo facilities ${ }^{14}$, as well as how much information visitors learn and understand ${ }^{14-16}$. Similarly, an online tool has been used to assess attitudes toward hypothetical experimental manipulations on pigs ${ }^{17}$. However, to our knowledge, there are no reports of attempts to measure the effect of science engagement activities on the attitudes of the audience toward neuroscientific research on animals.

As with many other human activities, the COVID-19 pandemic caused science engagement events to be moved online. This, in turn, brought new opportunities and advantages, as people from all over the world could now attend events that used to be restricted to locals and different types of interaction were facilitated: people could write their questions and discuss what was being presented at any time without needing to speak in public. Many of these events were recorded and then uploaded to video-sharing platforms, allowing for still more people to benefit from them. Moreover, researchers could share relevant links and documents with the audience and, importantly, create surveys to gauge their opinions.

We recently participated in two prominent public science festivals that were based in the UK but took place online: the IF Oxford Science and Ideas Festival 2020 and the British Neuroscience Association 'Bring Your Own Brain' 2021 Festival of Neuroscience. Our goal was to convey the rationale behind the use of NHPs in our neurophysiological and neuroimaging projects, which are aimed at elucidating the dynamics of cortico-thalamocortical interactions during learning, memory and decision-making, and understanding the effect of their disruption in neurological diseases. First, we explained the extensive regulatory and ethical approval that we must obtain to be authorized to carry out experimental procedures on NHPs in the UK. Then, we illustrated the different stages that the NHPs experience, from training to experimentation, showing real footage of the team members working with them. Importantly, we also highlighted our research on NHP welfare optimization and on the development of care refinements (for example, ref. $\left.{ }^{18}\right)$. Finally, we explained some of our recent research findings to highlight the value of our work (for example, ref. ${ }^{19}$ ). These 40 -min presentations were recorded and are freely available to view on YouTube ${ }^{20,21}$, meaning they can continue to have an effect. Overall, our social media videos ${ }^{20-22}$ have been viewed over 22,000 times.

Importantly, before we began our presentations, we asked participants ( $n=99)$ to voluntarily and anonymously answer two questions regarding their views on animal research (Fig. 1). Sixty-three participants completed this first couple of questions. These same questions were then repeated at the end of the event, plus an additional question that evaluated the effect of our presentation. Sixty-nine participants completed this second set of questions. In the beginning, most of the attendees (44\%) declared that they felt 'not very well informed' about animal research, and only $13 \%$ claimed to be 'very well informed'. These results mirror those by IPSOS Mori in the UK population (38 and $6 \%$, respectively) ${ }^{6}$. After our presentation, the great majority (59\%) felt that they were now 'fairly well informed', whereas 
those considering themselves uninformed dropped from an overall $57 \%$ to merely $17 \%$. Before our presentation, most people (71\%) (Fig. 1) already thought that 'animal research was necessary and could be done without cruelty' and only one person (1\%) thought that 'animals should never be used for research' This is in contrast with the 2018 IPSOS Mori poll that reported 38\% of people agreed with the statement: 'I think that animals should not be used in any scientific research because of the importance I place on animal welfare ${ }^{6}$. Perhaps this difference is due to our events appealing more to those already supportive of scientific research in animals. Another factor could be that the COVID-19 pandemic has caused an increase in approval for animal research, as shown by recent polls by Understanding Animal Research in the UK (https://www. understandinganimalresearch.org.uk/ $)^{23}$ and by the Foundation for Biomedical Research in the USA ${ }^{24}$. Critically, comparisons between different polls (for example, ref. ${ }^{25}$ ) also reveal that when people are given more context about the rationale behind the use of animals in research, they tend to oppose it less and instead declare themselves to be undecided. This is in accordance with our own findings: by the end of our presentation, the proportion who originally thought that 'animal research is necessary but cruel' decreased from $27 \%$ to $17 \%$, with individuals from this group who changed their mind now agreeing that 'animal research can be done without cruelty' or declaring themselves to be undecided.

Finally, perhaps the more straightforward proof that speaking openly is beneficial for both scientists and the public is that more than half of the attendees (Fig. 1) who participated in our polls thought that our presentation 'positively changed their perception of animal research'

Although it is evident that neuroscientists working with animals need to make their voices heard to achieve accurate representations of their work, it is also clear that we should pay attention to and address the concerns of the public. Results from polls and surveys such as ours suggest that when scientists are open about their work with NHPs, public support increases. Thus, the time is ripe for researchers to engage more globally and, crucially, to make use of technological advances to listen to and better engage their audiences, generating ongoing, mutually beneficial interactions.

\section{Data availability}

All data generated and analyzed in this study are included in this Comment.

\section{Juan Carlos Mendez (D) 1,2, Brook A. L. Perry (D)1, Rhyanne J. Heppenstall (D), Stuart Mason' and Anna S. Mitchell (D) $1 \square$ \\ ${ }^{1}$ Department of Experimental Psychology, University of Oxford, Oxford, UK. ${ }^{2}$ College of Medicine and Health, University of Exeter, Exeter, UK.

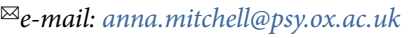

Published online: 3 March 2022

https://doi.org/10.1038/s41593-022-01039-z

References

1. Roelfsema, P. R. \& Treue, S. Neuron 82, 1200-1204 (2014).

2. MacArthur Clark, J., Clifford, P., Jarrett, W. \& Pekow, C. ILAR J. 60, 34-42 (2019)

3. Understanding Animal Research. $360^{\circ}$ Laboratory Animal Tours, https://www.labanimaltour.org/ (accessed February 2022).

4. Mitchell, A. S. et al. Neuroimage 229, 117700 (2021)

5. Homberg, J. R. et al. Neuron 109, 2374-2379 (2021)

6. Ipsos MORI. Public Attitudes to Animal Research in 2018 (Department for Business, Energy \& Industrial Strategy, 2018).

7. UK Government and Parliament. Ban Animal Testing - Fund, accept \& promote alternatives to animal testing. petition. parliament.uk, https://go.nature.com/3JyDXdW (October 2021).

8. Pew Research Center. Major gaps between the public, scientists on key issues. pewresearch.org, https://go.nature.com/3LJ38wf (1 July 2015).
9. The Federal Council. Popular initiative "Yes to the ban on animal and human experiments - Yes to research that brings safety and progress". admin.ch, https://go.nature.com/3GYVZUY (10 February 2022)

10. Bennett, A. J. \& Ringach, D. L. Neuron 92, 653-657 (2016).

11. Nat. Neurosci. 9, 1195 (2006).

12. Pham, D. NPJ Sci. Learn. 1, 16010 (2016).

13. Weingart, P., Joubert, M. \& Connoway, K. PLoS ONE 16, e0254201 (2021).

14. Waller, B. M., Peirce, K., Mitchell, H. \& Micheletta, J. PLoS ONE 7, e44680 (2012).

15. Whitehouse, J. et al. PLoS ONE 9, el13395 (2014).

16. Bowler, M. T., Buchanan-Smith, H. M. \& Whiten, A. PLoS ONE 7, e34505 (2012).

17. Schuppli, C. A., Molento, C. F. \& Weary, D. M. Public Underst. Sci. 24, 358-374 (2015).

18. Perry, B. A. L. et al. J. Neurosci. Methods 348, 108992 (2021).

19. Pelekanos, V. et al. J. Neurosci. 40, 7887-7901 (2020).

20. IF Oxford. Behind the scenes at a primate lab. youtube.com, https://youtu.be/ZO2NqgYZB7E (5 October 2020).

21. British Neuroscience Association. Behind the scenes of a primate lab. youtube.com, https://youtu.be/gCCXYAeNmRw (23 April 2021).

22. Wellcome Trust. Unravelling how the brain works. facebook.com, https://fb.watch/6bHEib6N7l/ (3 February 2019).

23. Williams, B. Public Attitudes to Animal Research Under COVID-19 (Understanding Animal Research, 2020).

24. Foundation for Biomedical Research. 2021 animal research poll. fbresearch.org, https://fbresearch.org/polls-2021/ (2021).

25. Speaking of Research. Unveiled: the moveable middle on animal research is larger than we thought. speakingofresearch.com, https://go.nature.com/34IAAm9 (16 June 2021).

\section{Acknowledgements}

These outreach activities were supported by the University of Oxford 'Enriching Engagement' scheme, funded by the Wellcome Trust. The Thalamus, Cortex, and Cognition Lab is funded by the Wellcome Trust (110157/Z/15/Z). We thank the organizers of the IF Oxford Science and Ideas Festival 2020 and the British Neuroscience Association 'Bring Your Own Brain' 2021 Festival of Neuroscience for hosting our online science engagement events. The University of Oxford Medical Sciences Interdivisional Research Ethics Committee (MS IDREC) indicated that formal ethical approval was not required as no personal information was collected in the survey responses.

\section{Competing interests}

The authors declare no competing interests.

\section{Additional information}

Peer review information Nature Neuroscience thanks Michele Basso for their contribution to the peer review of this work. 